\title{
RESEARCH OF STABILIZATION CONDITIONS AND ROBUST STABILITY OF DISCRETE ALMOST CONSERVATIVE SYSTEMS
}

\author{
Olena Teteriatnyk \\ Institute of Mathematics of NAS of Ukraine \\ 3 Tereschenkivska str., Kyiv, Ukraine, 301601 \\ E.Teteryatnik@gmail.com
}

\begin{abstract}
Conditions for the stabilizability of discrete almost conservative systems in which the coefficient matrix of a conservative part has no multiple eigenvalues are investigated.

It is known that a controllable system will be stabilized if its coefficient matrix is asymptotically stable.

The system stabilization algorithm is constructed on the basis of the solvability condition for the Lyapunov equation and the positive definiteness of $\mathrm{P}_{0}$ and $\mathrm{Q}_{1}$.

This theorem shows how to find the parameters of a controlled system under which it will be asymptotically stable for sufficiently small values of the parameter $\varepsilon(\mathrm{P}>0, \mathrm{Q}>0)$.

In addition, for a small parameter $\varepsilon$ that determines the almost conservatism of the system, an interval is found in which the conditions for its stabilizability are satisfied (Theorem 2).
\end{abstract}

Keywords: robust stability, almost conservative systems, Lyapunov equation.

\section{Introduction}

When developing modern navigation and gyroscopic devices for aircraft industry and shipping industry, controlled mathematical models are often used [1], which belong to the class of almost conservative systems. Recently, the search for analytical solutions in the construction of robust stable systems has come to the forefront of research. Therefore, finding in the analytical form of a robust controller for discrete almost conservative systems is topical.

In [2], the problem of stabilization of continuous almost conservative systems with a small parameter $\varepsilon>0$ is considered. Let's apply this approach to the stabilization of discrete almost conservative systems. The basis is the fact [3] that, under certain restrictions on the matrix coefficients of almost conservative systems, there exists $\varepsilon_{0}>0$, for which the parameter $\varepsilon$ from the interval $\left(0, \varepsilon_{0}\right)$ does not affect the stability of these systems.

\section{The aim and objectives of research}

To investigate the stabilizability conditions of discrete almost conservative systems in which the coefficient matrix of the conservative part has no multiple eigenvalues if the parameter is small enough.

To construct a robust controller for discrete almost conservative systems in the analytical form.

To expand the limits of the application of the method $[2,3]$. If necessary, to find additional conditions those ensure the symmetry of the matrix $\mathrm{P}_{0}$ in the case of discrete almost conservative systems.

To find the interval for parameter $\varepsilon$ for the closed system in which the stabilization conditions are satisfied.

\section{Construction of a stabilizing controller in analytical form}

Let's consider a discrete controlled almost conservative system

$$
\mathrm{x}(\mathrm{k}+1)=\left(\mathrm{F}_{0}+\varepsilon \mathrm{F}_{1}\right) \mathrm{x}(\mathrm{k})+\varepsilon \mathrm{G} \mathrm{u}(\mathrm{k}), \mathrm{x}(0)=\mathrm{x}_{0}, \mathrm{k}=0,1, \ldots,
$$

where $\mathrm{x} \in \mathfrak{R}_{\mathrm{n}}$ - the state vector, $\mathrm{F}_{0} \in \mathfrak{R}_{\mathrm{n} \times \mathrm{n}}$ - the orthogonal matrix $\left(\mathrm{F}_{0}^{\mathrm{T}} \mathrm{F}_{0}=\mathrm{F}_{0} \mathrm{~F}_{0}^{\mathrm{T}}=\mathrm{I}\right), \mathrm{F}_{1} \in \mathfrak{R}_{\mathrm{n} \times \mathrm{n}}-$ an arbitrary constant matrix, $\mathrm{u} \in \mathfrak{R}_{\mathrm{m}}$ - the control vector, $\mathrm{G} \in \mathfrak{R}_{\mathrm{n} \times \mathrm{m}}$ - the matrix for control, $\varepsilon>-$ a small parameter. 
Let's assume that the control of the system (1) depends linearly on its state

$$
\mathrm{u}(\mathrm{k})=\mathrm{Hx}(\mathrm{k}) \text {, }
$$

where $\mathrm{H} \in \mathfrak{R}_{\mathrm{m} \times \mathrm{n}}$ - some unknown constant matrix.

The closed system (1), (2) will be stabilizable if its coefficient matrix $F_{0}+\varepsilon\left(F_{1}+G H\right)$ is asymptotically stable, that is, it satisfies the Lyapunov matrix equation [4]

$$
\left[\mathrm{F}_{0}+\varepsilon\left(\mathrm{F}_{1}+\mathrm{GH}\right)\right]^{\mathrm{T}} \mathrm{P}\left[\mathrm{F}_{0}+\varepsilon\left(\mathrm{F}_{1}+\mathrm{GH}\right)\right]-\mathrm{P}=-2 \mathrm{Q},
$$

where $\mathrm{P}, \mathrm{Q} \in \mathfrak{R}_{\mathrm{n} \times \mathrm{n}}$ are positive definite matrices. Let's find the matrix $\mathrm{H}$ on the basis of a positive definite solution $\mathrm{P}, \mathrm{Q}$ of the reduced equation.

On the basis of the form of equation (3), let's find symmetric solution matrices P and Q in the form of power series in the small parameter

$$
\mathrm{P}=\mathrm{P}_{0}+\varepsilon \mathrm{P}_{1}+\varepsilon^{2} \mathrm{P}_{2}+\ldots, \mathrm{Q}=\mathrm{Q}_{0}+\varepsilon \mathrm{Q}_{1}+\varepsilon^{2} \mathrm{Q}_{2}+\ldots
$$

Let's assume that the conditions for the convergence of the series (4) [5] are satisfied. The matrix equation (3) is equivalent to an infinite system of equations $[3,6]$ :

$$
\mathrm{P}_{0}-\mathrm{F}_{0}^{\mathrm{T}} \mathrm{P}_{0} \mathrm{~F}_{0}=0
$$

$$
\begin{aligned}
& \mathrm{P}_{1}-\mathrm{F}_{0}^{\mathrm{T}} \mathrm{P}_{1} \mathrm{~F}_{0}=\mathrm{F}_{0}^{\mathrm{T}} \mathrm{P}_{0}\left(\mathrm{~F}_{1}+\mathrm{GH}\right)+\left(\mathrm{F}_{1}+\mathrm{GH}\right)^{\mathrm{T}} \mathrm{P}_{0} \mathrm{~F}_{0}+2 \mathrm{Q}_{1}, \\
& \mathrm{P}_{2}-\mathrm{F}_{0}^{\mathrm{T}} \mathrm{P}_{2} \mathrm{~F}_{0}=\mathrm{F}_{0}^{\mathrm{T}} \mathrm{P}_{1}\left(\mathrm{~F}_{1}+\mathrm{GH}\right)+\left(\mathrm{F}_{1}+\mathrm{GH}\right)^{\mathrm{T}} \mathrm{P}_{1} \mathrm{~F}_{0}+\left(\mathrm{F}_{1}+\mathrm{GH}\right)^{\mathrm{T}} \mathrm{P}_{0}\left(\mathrm{~F}_{1}+\mathrm{GH}\right)+2 \mathrm{Q}_{2},
\end{aligned}
$$$$
\mathrm{P}_{\mathrm{i}}-\mathrm{F}_{0}^{\mathrm{T}} \mathrm{P}_{\mathrm{i}} \mathrm{F}_{0}=\mathrm{F}_{0}^{\mathrm{T}} \mathrm{P}_{\mathrm{i}-1}\left(\mathrm{~F}_{1}+\mathrm{GH}\right)+\left(\mathrm{F}_{1}+\mathrm{GH}\right)^{\mathrm{T}} \mathrm{P}_{\mathrm{i}-1} \mathrm{~F}_{0}+\left(\mathrm{F}_{1}+\mathrm{GH}\right)^{\mathrm{T}} \mathrm{P}_{\mathrm{i}-2}\left(\mathrm{~F}_{1}+\mathrm{GH}\right)+2 \mathrm{Q}_{\mathrm{i}},
$$

Equation (5) shows the commutativity of the matrices $\mathrm{F}_{0}, \mathrm{P}_{0}$. Let's assume that the orthogonal matrix $\mathrm{F}_{0}$ does not have multiple eigenvalues. In this case, the matrix $\mathrm{P}_{0}$ can be represented in the form [7]

$$
\mathrm{P}_{0}=\alpha_{0} \mathrm{I}_{\mathrm{n}}+\alpha_{1} \mathrm{~F}_{0}+\ldots+\alpha_{\mathrm{n}-1} \mathrm{~F}_{0}^{\mathrm{n}-1}
$$

where $\alpha_{0}, \alpha_{1}, \ldots, \alpha_{n-1}$ are the free parameters. $I_{n}$ is the identity matrix of dimension $n$. In contrast to the continuous case, the right-hand side of equation (7) is not symmetric, so the problem arises to ensure not only its positive definiteness, but also symmetry, which can be done with the help of free parameters. in the form

Using some non-degenerate orthogonal transformation $U \in \Re_{\mathrm{n} \times \mathrm{n}}$, the matrix $\mathrm{F}_{0}$ can be written

$$
\mathrm{F}_{0}=\mathrm{UF}_{0} \mathrm{U}^{\mathrm{T}}=\operatorname{Udiag}\left\{\mathrm{W}_{1}, \ldots, \mathrm{W}_{\mathrm{r}}\right\} \mathrm{U}^{\mathrm{T}},
$$

where $W_{1}=-W_{2}=1, W_{i}=\left[\begin{array}{cc}a_{i} & b_{i} \\ -b_{i} & a_{i}\end{array}\right], a_{i}^{2}+b_{i}^{2}=1, b_{i}^{2} \neq b_{j}^{2}, i \neq j, i, j \in\{\overline{3, r}\}, 2 r=n+2$.

Let's multiply the i-th equation of the system (6) from the left by $\mathrm{U}^{\mathrm{T}}$ and from the right by U and obtain

$$
\tilde{\mathrm{P}}_{\mathrm{i}}-\tilde{\mathrm{F}}_{0}^{\mathrm{T}} \tilde{\mathrm{P}}_{\mathrm{i}} \tilde{\mathrm{F}}_{0}=\tilde{\mathrm{D}}_{\mathrm{i}}, \mathrm{i}=1,2, \ldots,
$$


where, $\tilde{P}_{i}=U^{T} P_{i} U, \tilde{D}_{i}=\left\{\tilde{d}_{t q}^{i}\right\}_{1}^{n}=U^{T} D_{i} U$, and $D_{i}$ is the right-hand side of the initial equation. In (9) it is taken into account that

$$
\mathrm{U}^{\mathrm{T}} \mathrm{F}_{0}^{\mathrm{T}} \mathrm{U}=\left(\mathrm{U}^{\mathrm{T}} \mathrm{F}_{0} \mathrm{U}\right)^{\mathrm{T}}
$$

In [8], the solution of the Lyapunov matrix equation for discrete almost conservative systems is given, when the orthogonal matrix has the canonical form (8). It is shown there that the elements of the matrix $\tilde{D}_{i}$ must satisfy the following conditions for the solvability of equations (9):

$$
\tilde{\mathrm{d}}_{11}^{\mathrm{i}}=\tilde{\mathrm{d}}_{22}^{\mathrm{i}}=0, \tilde{\mathrm{d}}_{\mathrm{jj}}^{\mathrm{i}}+\tilde{\mathrm{d}}_{\mathrm{j}+1, \mathrm{j}+1}^{\mathrm{i}}=0, \mathrm{j}=3,5, \ldots, \mathrm{n}-1, \mathrm{i}=1,2, \ldots
$$

Thus, the right-hand side of equation (9) must satisfy $r$ conditions and the same number of free parameters remains in the matrix $\tilde{\mathrm{P}}_{\mathrm{i}}$ after its calculation from the i-th equation. Let's assume that the matrices $\mathrm{Q}_{\mathrm{i}}, \mathrm{i}=1,2, \ldots$ are given in a specific or parametric form, but so that the calculated matrix $Q$ is positive definite.

The transition to the canonical form can be done with the help of a non-degenerate orthogonal transformation, therefore the solvability of equations (6) is also involved some $r$ conditions with respect to the right-hand side of these equations. On the other hand, after solving such equation, not all parameters of the matrix $P_{i}$ will get specific values, but free parameters will remain. They will get values at the (i+1)-th step, similarly as in [9], or at subsequent steps when conditions are agreed.

It is known [10] that a linear matrix equation has a solution when the rank of the coefficient matrix is equal to the rank of the extended matrix. Let's find similar conditions to which the right-hand side of equations (6) must correspond so that they have a solution. To do this, let's pass to the equivalent equation, which coefficient matrix has the size $n^{2}$. This can be done through a direct product [11].

Since the right side of $\mathrm{i}$-th equation system (6) is denoted by $\mathrm{D}_{\mathrm{i}}$, then 1-th rows respectively $\mathrm{D}_{\mathrm{i}}$, Pi matrix are denoted by $\mathrm{D}_{\mathrm{i}, 1 *}, \mathrm{P}_{\mathrm{i}, 1 *}$. Let's obtain the following equivalent system of equations:

$$
\breve{\mathrm{F}} \theta_{\mathrm{i}}=\xi_{\mathrm{i}}, \mathrm{i}=1,2, \ldots
$$

where $\breve{F}=I_{n} \otimes I_{n}-F_{0}^{T} \otimes F_{0}^{T}, \theta_{i}=\left[P_{i, 1 *}, \ldots, P_{i, n *}\right]^{T}, \xi_{i}=\left[D_{i, 1 *}, \ldots, D_{i, n *}\right]^{T}, \breve{F} \in \Re_{n^{2} \times n^{2}} \otimes$ is the symbol of the direct product. The symmetry of the matrix $\mathrm{P}_{i}$ with the solution $\theta_{i}$ is reached by means of free parameters.

So, if the equalities (necessary and sufficient conditions) are satisfied,

$$
\operatorname{rank} \breve{\mathrm{F}}=\operatorname{rank}\left[\breve{\mathrm{F}}, \xi_{\mathrm{i}}\right], \mathrm{i}=1,2, \ldots,
$$

then equations (11) are solvable. In other words, the matrices of both sides of (12) have a common zero-space.

The matrix $\mathrm{F}_{0}^{\mathrm{T}} \otimes \mathrm{F}_{0}^{\mathrm{T}}$ has eigenvalues $\lambda_{1} \lambda_{\mathrm{j}}[11]$, where $\lambda_{1}, \lambda_{\mathrm{j}}, 1, \mathrm{j} \in\{\overline{1, \mathrm{n}}\}$ are the eigenvalues of the matrix $F_{0}$, that is, the $n$ eigenvalues of the matrix $F$ are zero because $\lambda_{1} \bar{\lambda}_{1}=1,1 \in\{\overline{1, n}\}$. $\bar{\lambda}_{1}$ are complex conjugate eigenvalues. Let's the vector $\gamma \in \mathfrak{R}_{\mathrm{n}^{2}}$ is a general solution of the system of equations

$$
\breve{F} \gamma=0
$$

The vector $\gamma$ describes the zero-space of the matrix $\breve{F}$. From the properties of the direct product it follows that the matrix $\mathrm{F}_{0}^{\mathrm{T}} \otimes \mathrm{F}_{0}^{\mathrm{T}}$ is orthogonal. Then let's obtain

$$
\left(\mathrm{F}_{0}^{\mathrm{T}} \otimes \mathrm{F}_{0}^{\mathrm{T}}\right)(\breve{\mathrm{F}})^{\mathrm{T}} \gamma=\left(\mathrm{F}_{0}^{\mathrm{T}} \otimes \mathrm{F}_{0}^{\mathrm{T}}-\mathrm{I}_{\mathrm{n}} \otimes \mathrm{I}_{\mathrm{n}}\right)=-\breve{\mathrm{F}} \gamma=0 .
$$

In this case $(\breve{\mathrm{F}})^{\mathrm{T}} \gamma=0$. If multiply both sides of equations (11) on the left by a non-zero transposed vector $\gamma^{T}$ that has arbitrary constants, then obtain zero identities if and only if the equations have solutions. This follows from the properties of the vector. 
Thus, the free parameters of the matrix $\mathrm{P}_{\mathrm{i}-1}, \mathrm{i}=1,2, \ldots$, can be determined from the condition for the solvability of the i-th equation, namely,

$$
\left(\gamma, \xi_{\mathrm{i}}\right)=0
$$

To perform (14) and calculate the free parameters of the matrix $\mathrm{P}_{\mathrm{i}-1}$ in the scalar pro$\operatorname{duct}\left(\gamma, \xi_{\mathrm{i}}\right)$, it is necessary to equate the coefficients for arbitrary constants to zero.

Now let's calculate the matrix $\mathrm{P}_{\mathrm{i}}$ with free parameters. First, let's reduce the matrix $\breve{\mathrm{F}}$ to the upper triangular form by means of left elementary operations on polynomial matrices [10], which correspond to the matrices $\mathrm{S}_{1} \mathrm{~S}_{2}, \ldots, \mathrm{S}_{1}$. Thus, the transformation matrix $\mathrm{S}=\mathrm{S}_{1} \mathrm{~S}_{1-1} \ldots \mathrm{S}_{1}$ allows to proceed to a simplified system of equations

$$
\mathrm{SF} \theta_{\mathrm{i}}=\mathrm{S} \xi_{\mathrm{i}}, \mathrm{i}=1,2, \ldots
$$

From equations (15), the vector $\theta_{i}$ is found quite simply by calculating unknown elements from the bottom up. The symmetry of the matrix $\mathrm{P}_{\mathrm{i}}$ is coordinated by means of a part with $\mathrm{n}$ free parameters.

It should be noted that the separation of procedures for finding the matrix $\mathrm{P}_{\mathrm{i}}$ with free parameters and the specific values of the free parameters of the matrix $\mathrm{P}_{\mathrm{i}-1}$ leads to a simplification of calculations and identification of internal relationships of the equation that can be used to solve other problems, for example, the problem of system stabilization.

Let's describe the stabilization of the system (1), (2), based on condition (14) and a positive definite solution of the Lyapunov matrix equation (3).

Theorem 1. Let's suppose that a general orthogonal matrix $\mathrm{F}_{0}$ of the system (1) does not have multiple eigenvalues, and $\mathrm{Q}_{1}$ is a symmetric positive definite matrix.

Then, if the elements of the matrix $\mathrm{H}$ and the expansion coefficients (7) satisfy the following conditions:

$$
\begin{gathered}
\left(\gamma, \xi_{1}\right)=0, \\
\mathrm{P}_{0}^{\mathrm{T}}=\mathrm{P}_{0}
\end{gathered}
$$

and one of the alternatives

$$
\alpha_{0}>\max _{\mathrm{i}}\left\{\sum_{\mathrm{j}=1(\mathrm{j} \neq \mathrm{i})}^{\mathrm{n}}\left|\mathrm{v}_{\mathrm{ij}}\right|-\mathrm{v}_{\mathrm{ii}}\right\}, \mathrm{i}=\overline{1, \mathrm{n}}
$$

or

$$
\alpha_{0}>-\lambda_{\min }(\mathrm{V})
$$

where $\lambda_{\text {min }}(V)$ is the minimal eigenvalue of the matrix $V$,

$$
V=\left\{v_{i j}\right\}=\left\{\begin{array}{cc}
0, & \text { if } n=1 \\
\alpha_{1} F_{0}+\ldots+\alpha_{n-1} F_{0}^{n-1}, & \text { if } n \geq 2
\end{array}\right.
$$

then the closed system (1), (2) is a stabilized.

Proof. By the hypothesis of the theorem, the orthogonal matrix $\mathrm{F}_{0}$ does not have multiple eigenvalues; therefore, the zero approximation of the matrix-solution can be written in the form of a polynomial in the permutation matrix (7). Equality (17) shows the symmetry of the matrix $\mathrm{P}_{0}$, which is always achieved with the parameters $\alpha_{1}, \ldots, \alpha_{\mathrm{n}-1}$.

Let's show that the conditions (18), (19) are correct. The zero approximation $P_{0}=\left\{p_{i j}\right\}_{1}^{n}$ of the solution matrix $\mathrm{P}$ must be a positive definite matrix, because in the stabilized closed sys- 
tem (1), (2) the matrix of coefficients is asymptotically stable. The conditions for positive definiteness follow from Gershgorin theorem [12]

$$
\mathrm{p}_{\mathrm{ii}}=\sum_{\mathrm{j}=1(\mathrm{j} \neq \mathrm{i})}^{\mathrm{n}}\left|\mathrm{p}_{\mathrm{ij}}\right|, \mathrm{i}=\overline{1, \mathrm{n}} .
$$

By analogy with (20), a sufficient condition for the matrix $\mathrm{P}_{0}=\alpha_{0} \mathrm{I}+\mathrm{V}$ can be represented in terms of the dominant diagonal $\alpha_{0} \mathrm{I}$, namely, inequality (18).

To obtain a positive definite matrix $\mathrm{P}_{0}$, the condition on the parameter $\alpha_{0}$ can be represented in another way. It is known [10] that the eigenvalues of a positive definite matrix are all positive, that is,

$$
\lambda\left(\mathrm{P}_{0}\right)=\lambda\left(\alpha_{0} \mathrm{I}+\mathrm{V}\right)=\alpha_{0}+\lambda(\mathrm{V})>0,
$$

where $\lambda(\cdot)$ is an arbitrary eigenvalue of the matrix. From the inequality (21) obtain the condition of positive definiteness of the matrix $\mathrm{P}_{0}(19)$, which defines the exact lower bound for the parameter $\alpha_{0}$, while condition (18) can determine the overestimated one.

From the above (equations (11)-(14)) it follows that condition (16) determines the solvability of the first equation of system (6). The vector $\gamma$ has arbitrary constants; therefore, to determine the required parameters, it is sufficient to equate the coefficients of these constants with zero, taking into account conditions (17)-(19). If it can achieve the fulfillment of (16) with the help of the parameters of the matrix $\mathrm{H}$, than let's calculate the concrete symmetric positive definite matrix $\mathrm{P}_{0}$ from the conditions (17)-(19), and then let's find the matrix H from (16).

Thus, the matrix $\mathrm{P}_{0}>0$ is constructed and $\mathrm{Q}_{1}>0$ is chosen, therefore, in accordance with the expansions (4), the matrices $P_{i}, Q_{i+1}, i=1,2, \ldots$ do not affect the positive definiteness of the matrices $\mathrm{P}, \mathrm{Q}$, respectively. So, if the matrix $\mathrm{H}$ satisfies equality (16), then the matrix of coefficients $\mathrm{F}_{0}+\varepsilon$ $\left(\mathrm{F}_{1}+\mathrm{GH}\right)$ of the closed system (1), (2) will be asymptotically stable.

It should be noted that the formulas (16), (19) are more suitable for the search for the matrix $\mathrm{H}$, when the data are given in numerical form, and (16), (18) - in symbolic and (or) numerical.

Let's illustrate the results of Theorem 1 with a simple example.

Example 1. Let the system (1), (2) be given with the following parameters:

$$
\begin{gathered}
\mathrm{F}_{0}=\left[\begin{array}{cccc}
0 & 1 / 2 & 1 / 2 & 1 / 2 \sqrt{2} \\
0 & 1 / 2 & 1 / 2 & -1 / 2 \sqrt{2} \\
-1 / 2 \sqrt{2} & -1 / 2 & 1 / 2 & 0 \\
-1 / 2 \sqrt{2} & 1 / 2 & -1 / 2 & 0
\end{array}\right], F_{1}=\left[\begin{array}{cccc}
2 & -1 & 0 & 0 \\
0 & 0 & 1 & 0 \\
0 & -2 & -1 & 0 \\
0 & 0 & -1 & 4
\end{array}\right], \mathrm{G}^{\mathrm{T}}=\left[\begin{array}{cccc}
0 & 1 & 0 & 0 \\
0 & 0 & 0 & 1
\end{array}\right], \\
\mathrm{F}_{0}^{\mathrm{T}} \mathrm{F}_{0}=\mathrm{F}_{0} \mathrm{~F}_{0}^{\mathrm{T}}=\mathrm{I}, \mathrm{H} \in \mathfrak{R}_{2 \times 4} .
\end{gathered}
$$

It is necessary to stabilize the given discrete almost conservative system.

The matrix $\mathrm{F}_{0}$ has different eigenvalues:

$$
\begin{gathered}
\lambda_{1,2}=-0.11840644 \pm 0.99296521 \mathrm{i}, \\
\lambda_{3,4}=-0.61840644 \pm 0.78585843 \mathrm{i},\left|\lambda_{\mathrm{i}}\right|=1, \quad \mathrm{i}=\overline{1,4}
\end{gathered}
$$

therefore, to stabilize a given system, let's apply Theorem 1. The zero approximation of the solution $\mathrm{P}$ has the form

$$
\mathrm{P}_{0}=\alpha_{0} \mathrm{I}_{3}+\alpha_{1} \mathrm{~F}_{0}+\alpha_{2} \mathrm{~F}_{0}^{2}+\alpha_{3} \mathrm{~F}_{0}^{3}
$$


The condition $\mathrm{P}_{0}=\mathrm{P}_{0}^{\mathrm{T}}$ implies that $\alpha_{2}=-\alpha_{3}, \alpha_{1}=(1 / \sqrt{2}) \alpha_{3}$. Let $\alpha_{3}=1$, then the minimal eigen value of the matrix $\mathrm{V}=\alpha_{3}\left((1 / \sqrt{2}) \mathrm{F}_{0}-\mathrm{F}_{0}^{2}+\mathrm{F}_{0}^{3}\right)$ is equal to $\lambda_{\min }(\mathrm{V})=1 / 2-(\sqrt{5-2 \sqrt{2}}) / 2$, hence from (19) let's choose $\alpha_{0}=2>-\lambda_{\min }(\mathrm{V})$ and obtain a positive definite matrix $\mathrm{P}_{0}$.

Since equality $\operatorname{rank}\left[\mathrm{I}_{4} \otimes \mathrm{I}_{4}-\mathrm{F}_{0}^{\mathrm{T}} \otimes \mathrm{F}_{0}^{\mathrm{T}}\right]=12$ holds, the vector $\gamma$ has free parameters, there are four of them: $v_{1}, v_{2}, v_{3}, v_{4}$. Let's select the matrix $Q_{1}=I$ and form the vector $\xi_{1} \in \Re_{16}$ from the elements of the matrix $\mathrm{D}_{1}$ along the rows from top to bottom (11). In equation (16) let's equate to zero the coefficients for arbitrary constants of the vector $\gamma$ and from the system of equations, two of which are independent, let's obtain the elements of the required matrix

$$
\mathrm{H}=\left[\begin{array}{cccc}
-\frac{606}{49}-\frac{204}{49} \sqrt{2} & 0 & 0 & -\frac{20}{49}+\frac{149}{49} \sqrt{2} \\
0 & 0 & 0 & 0
\end{array}\right]
$$

The free parameters in (22) are set equal to zero.

Thus, the asymptotically stable matrix of the coefficients $\mathrm{F}=\mathrm{F}_{0}+\varepsilon\left(\mathrm{F}_{1}+\mathrm{GH}\right)$ of the closed system (1), (2) has the form

$$
\mathrm{F}=\left[\begin{array}{cccc}
2 \varepsilon & \frac{1}{2}-\varepsilon & \frac{1}{2} & \frac{1}{2 \sqrt{2}} \\
\varepsilon\left(-\frac{606}{49}-\frac{204}{49} \sqrt{2}\right) & \frac{1}{2} & \frac{1}{2}+\varepsilon & -\frac{\sqrt{2}}{2}+\varepsilon\left(-\frac{20}{49}+\frac{149}{49} \sqrt{2}\right) \\
-\frac{1}{2 \sqrt{2}} & -\frac{1}{2}-2 \varepsilon & \frac{1}{2}-\varepsilon & 0 \\
-\frac{1}{2 \sqrt{2}} & 1 / 2 & -\frac{1}{2}-\varepsilon & 4 \varepsilon
\end{array}\right]
$$

where the parameter $\varepsilon$ has sufficiently small values.

\section{Construction of robust stability interval}

Theorem 1 shows how to find the parameters of the system (1), (2) under which it will be asymptotically stable for sufficiently small values of the parameter $\varepsilon$. But it is expedient to find an interval for $\varepsilon$ in which the constructed closed system is asymptotically stable. For this, on the basis of Theorem 1, it is necessary to find a solution of the Lyapunov matrix equation (3) in the expansion (4) and an interval for the parameter $\varepsilon$ in which the solution matrices P and Q are positive definite.

The following statement shows how to do this.

Theorem 2. Let's suppose that the orthogonal matrix $\mathrm{F}_{0}$ of general form does not have multiple eigenvalues, the matrices $\mathrm{P}_{0}>0, \mathrm{Q}_{1}>0, \mathrm{H}$ are defined by Theorem 1 and the symmetric matrices

$$
\mathrm{P}=\mathrm{P}_{0}+\varepsilon \mathrm{P}_{1}, \mathrm{Q}=\varepsilon \mathrm{Q}_{1}+\varepsilon^{2} \mathrm{Q}_{2}+\varepsilon^{3} \mathrm{Q}_{3}
$$

satisfy the Lyapunov matrix equation (3).

Also, let $\mu_{\max }$ be the maximal eigenvalue of the pencil of matrices $\mu \mathrm{P}_{0}+\mathrm{P}_{1}, \delta_{\mathrm{i}}(\mathrm{i}=\overline{1,2 \mathrm{n}})$ are the eigenvalues of the quadratic matrix pencil $\delta^{2} \mathrm{Q}_{1}+\delta \mathrm{Q}_{2}+\mathrm{Q}_{3}$ and $\delta_{\min }, \delta_{\max }$ - respectively, its minimum and maximum real eigenvalues.

Then for arbitrary $\varepsilon \in \mathrm{r}_{1}(\mathrm{P}) \cap \mathrm{r}_{2}(\mathrm{P})$, where

$$
r_{1}(P)= \begin{cases}\left(0, \mu_{\max }^{-1}\right), & \text { if } \mu_{\max }>0 \\ (0,+\infty), & \text { if } \mu_{\max } \leq 0\end{cases}
$$




$$
\mathrm{r}_{2}(\mathrm{Q})=\left\{\begin{array}{cc}
\left(0, \delta_{\max }^{-1}\right) \cup\left(\delta_{\min }^{-1},+\infty\right), & \text { if } \delta_{\min }, \delta_{\max }>0 ; \\
\left(0, \delta_{\max }^{-1}\right), & \text { if } \delta_{\min } \leq 0, \delta_{\max }>0 \\
(0,+\infty), & \text { if } \delta_{\text {min }}, \delta_{\max } \leq 0 \\
& \text { or } \delta_{\mathrm{i}}(\mathrm{i}=\overline{1,2 \mathrm{n}})-\text { complex }
\end{array}\right.
$$

the matrices P, Q are positive definite.

Proof. Let's show that the solution matrices (23) exist.

The elements of the matrix $H$ and the coefficients $\alpha_{0}, \alpha_{1}, \ldots, \alpha_{n-1}$ are calculated from conditions (16)-(19) under the assumption of positive definiteness of the chosen symmetric matrix $\mathrm{Q}_{1}$. Let's find the matrix $\mathrm{P}_{0}$ from the expansion (7), and conditions (17)-(19) show its symmetry and positive definiteness.

Next, let's calculate the matrix $\mathrm{P}_{1}$ from the first equation of system (6) for the known righthand side $\mathrm{D}_{1}$, the condition for its resolution (16) is satisfied by Theorem 1 . The free parameters of the found matrix $\mathrm{P}_{1}$ can be set equal to zero or calculated, since this better inscribes it into the expansion (7) and, possibly, will give a wider range. To calculate the free parameters, let's choose the symmetric matrix $Q_{2}$ and equate the coefficients for arbitrary constants in the scalar product $\left(\gamma, \xi_{2}\right)$. In this case, the matrix $\mathrm{Q}_{2}$ is chosen only for calculation of the values of the free parameters. Parameters that do not take values are assumed to be zero.

Let's equate the matrices $P_{i}, Q_{i+2}, i=2,3$..to zero and $Q_{2}, Q_{3}$ let's calculate by the formulas:

$$
\begin{gathered}
\mathrm{Q}_{2}=-\left[\mathrm{F}_{0}^{\mathrm{T}} \mathrm{P}_{1}\left(\mathrm{~F}_{1}+\mathrm{GH}\right)+\left(\mathrm{F}_{1}+\mathrm{GH}\right)^{\mathrm{T}} \mathrm{P}_{1} \mathrm{~F}_{0}+\left(\mathrm{F}_{1}+\mathrm{GH}\right)^{\mathrm{T}} \mathrm{P}_{0}\left(\mathrm{~F}_{1}+\mathrm{GH}\right)\right] / 2, \\
\mathrm{Q}_{3}=-\left[\left(\mathrm{F}_{1}+\mathrm{GH}\right)^{\mathrm{T}} \mathrm{P}_{1}\left(\mathrm{~F}_{1}+\mathrm{GH}\right)\right] / 2 .
\end{gathered}
$$

This way of determining the elements of the expansions (4) gives grounds to state that the matrices $\mathrm{P}=\mathrm{P}_{0}+\varepsilon \mathrm{P}_{1}, \mathrm{Q}=\varepsilon \mathrm{Q}_{1}+\varepsilon^{2} \mathrm{Q}_{2}+\varepsilon^{3} \mathrm{Q}_{3}$ satisfy the Lyapunov matrix equation (3).

Now let's find conditions on the parameter $\varepsilon$ for which the matrices $\mathrm{P}, \mathrm{Q}$ are positive definite. Instead of the pencil $\mathrm{P}_{0}+\varepsilon \mathrm{P}_{1}$, let's consider the equivalent pencil of matrices $\mu \mathrm{P}_{0}+\mathrm{P}_{1}$, which has the same range of parameters $\varepsilon=1 / \mu$. The $\mathrm{P}_{0}, \mathrm{P}_{1}$ are symmetric, therefore the eigenvalues of the pencil $\mathrm{P}(\mu)$ are real numbers. $\mathrm{P}_{0}>0$, therefore, for sufficiently large values of $\mu>0$ the matrix $\mu \mathrm{P}_{0}+\mathrm{P}_{1}$ is positive definite, which follows from the eigenvalues of the matrix $\mu \mathrm{P}_{0}$ (they are large positive) and the eigenvalues of the sum of Hermitian (symmetric) matrices [10]. The eigenvalues of the matrix depend continuously on its elements [12], therefore their sign does not change to the first zero $\left|\mu \mathrm{P}_{0}+\mathrm{P}_{1}\right|$ right on the $\mu$-axis.

Thus, for $\mu>\mu_{\max }>0$, where $\mu_{\max }$ is the maximum eigenvalue of the pencil, have $\mu \mathrm{P}_{0}+\mathrm{P}_{1}>0$. If $\mu_{\max } \leq 0$, then let's obtain the interval $(0, \infty)$. The possible cases describe the intervals $(24)$.

Now let's consider the quadratic pencil of matrices $Q_{1}+\varepsilon Q_{2}+\varepsilon^{2} Q_{3}$. The matrix $Q_{1}$ is symmetric positive defined, therefore the given pencil of matrices is strictly equivalent to such pencil [10]:

$$
\mathrm{I}_{\mathrm{n}}+\varepsilon \mathrm{Q}_{1}^{-1 / 2} \mathrm{Q}_{2} \mathrm{Q}_{1}^{-1 / 2}+\varepsilon^{2} \mathrm{Q}_{1}^{-1 / 2} \mathrm{Q}_{3} \mathrm{Q}_{1}^{-1 / 2} .
$$

Let's set $\delta=\varepsilon^{-1}$ and pass to a pencil $\mathrm{Q}(\delta)=\delta^{2} \mathrm{I}_{\mathrm{n}}+\delta \mathrm{Q}_{1}^{-1 / 2} \mathrm{Q}_{2} \mathrm{Q}_{1}^{-1 / 2}+\mathrm{Q}_{1}^{-1 / 2} \mathrm{Q}_{3} \mathrm{Q}_{1}^{-1 / 2}$ that has the same range of values with the pencil $\delta^{2} \mathrm{Q}_{1}+\delta \mathrm{Q}_{2}+\mathrm{Q}_{3}$, and also with the initial sheaf of matrices for the parameter $\varepsilon=\delta^{-1}$. For sufficiently large values (including negative values) of the parameter $\delta$, the matrix $\mathrm{Q}(\delta)$ is positive definite, as a matrix with dominant diagonal elements. Moreover, if all the eigenvalues of the quadratic matrix pencil are complex, then the positive definiteness of Q $(\delta)$ does not depend on the parameter $\delta$. Taking into account that the eigenvalues of the matrix depend continuously on its elements, the positive definiteness of the matrix $\mathrm{Q}(\delta)$ does not change to the 
first zeros in $\mathrm{Q}(\delta) \mid$ right and left on the $\delta$-axis. Let's suppose that among the eigenvalues $\delta_{\mathrm{i}}, \mathrm{i}=\overline{1,2 \mathrm{n}}$ of a quadratic pencil of matrices $\mathrm{Q}(\delta)$ are real, and $\delta_{\min }, \delta_{\max }$ are respectively the minimal and the maximum real values. Let's consider the possible cases for the parameter $\varepsilon=\delta^{-1}$, when the matrix $Q$ is positive definite.

1. If all real eigenvalues are positive, then the parameter $\delta$ belongs to the intervals $0<\delta<\delta_{\text {min }}$, $\delta_{\max }<\delta<+\infty$. Then $\varepsilon=\delta^{-1}$ lies in such boundaries: $0<\varepsilon<\delta_{\max }^{-1}, \delta_{\min }^{-1}<\varepsilon<+\infty$.

2. If $\delta_{\min } \leq 0, \delta_{\max }>0$, then $\delta$ belongs to the interval $\delta_{\max }<\delta<+\infty$. Let's pass to the parameter $\varepsilon=\delta^{-1}$, and obtain the following interval: $0<\varepsilon<\delta_{\max }^{-1}$.

3. If all the real eigenvalues are non-positive, then the parameter, $\delta>0$, can be arbitrary, hence $0<\varepsilon<+\infty$.

The cases considered for the parameter $\varepsilon$ describe the intervals (26).

The intersection of the intervals constructed for the pencils of matrices $\mu \mathrm{P}_{0}+\mathrm{P}_{1}, \delta{ }^{2} \mathrm{Q}_{0}+\delta$ $\mathrm{Q}_{1}+\mathrm{Q}_{2}$ gives the required interval.

Let's continue the consideration of Example 1.

Example 2. Let's find the interval for the parameter $\varepsilon$ in which the coefficient matrix of the closed system (1), (2) found in Example 1 will be asymptotically stable.

From the first equation of system (6) for a known right-hand side let's find the matrix $\mathrm{P}_{1}$, and by formula (26) let's calculate the matrices $\mathrm{Q}_{2}, \mathrm{Q}_{3}$.

Next, let's find the maximal real eigenvalues of the pencils of the matrices $\mu \mathrm{P}_{0}+\mathrm{P}_{1}$ and $\delta^{2} \mathrm{Q}_{1}+\delta \mathrm{Q}_{2}+\mathrm{Q}_{3}$ :

$$
\begin{gathered}
\left|\mu \mathrm{P}_{0}+\mathrm{P}_{1}\right|=32.57106780 \mu^{4}-184.4561214 \mu^{3}+4471.144361 \mu^{2}+14659.80205 \mu+151731.5746 \\
\left|\delta^{2} \mathrm{Q}_{1}+\delta \mathrm{Q}_{2}+\mathrm{Q}_{3}\right|=\delta^{8}-198.7938233 \delta^{7}-14287.45132 \delta^{6}++1471614.476 \delta^{5}-22742729.35 \delta^{4}- \\
-11044054.80 \delta^{3}+205046627.4 \delta^{2}+107987709.2 \delta+103735989.2 \\
\mu_{\max }=10.83819977, \delta_{\max }=234.7151043
\end{gathered}
$$

The minimum real eigenvalue of a quadratic pencil is negative.

Thus, in the intervals $\left(0, \mu_{\max }^{-1}\right)$ and $\left(0, \delta_{\max }^{-1}\right)$, correspondingly, the $\mathrm{P}_{0}+\varepsilon \mathrm{P}_{1}, \mathrm{Q}_{1}+\varepsilon \mathrm{Q}_{2}+\varepsilon^{2} \mathrm{Q}_{3}$ are positive definite. Their common interval $\left(0, \delta_{\max }^{-1}=0.004260484228\right)$ for the parameter $\varepsilon$ gives an asymptotically stable coefficient matrix of the closed system.

It should be noted that the interval for $\varepsilon$, found from the sufficient conditions (24), (25), can significantly differ from the real interval in which the matrix $\mathrm{F}_{0}+\varepsilon\left(\mathrm{F}_{1}+\mathrm{GH}\right.$ is asymptotically stable. Especially, as a rule, the condition of positive definiteness of the matrix $Q_{1}+\varepsilon Q_{2}+\varepsilon^{2} Q_{3}$ narrows the interval. For example, for $\varepsilon=0.01$, the matrix of the closed system (1), (2) has such eigenvalues:

$$
\begin{aligned}
& \lambda_{1,2}=-0.06041715 \pm 0.99790688 \mathrm{i}, \\
& \lambda_{3,4}=0.58541715 \pm 0.80423321 \mathrm{i},
\end{aligned}
$$

which moduli are less than one, that is, the system is stabilized.

\section{Conclusions}

This work has fully disclosed the tasks. Conditions for the stabilizability of discrete almost conservative systems are found using the Lyapunov matrix equation. The presence of a small parameter in the matrix of coefficients makes it possible to simplify the solution in an analytical form, which can be used in subsequent work. Developed robust controller in analytical form for discrete almost conservative systems makes it possible to apply software in solving practical problems.

An interval is found for an unknown small parameter $\varepsilon$ in which a given closed system is asymptotically stable. 
The obtained results are an extension of the scope of described method. In the future, it is planned to systematize and generalize the main mechanical characteristics such as stabilization, stability and optimal control for discrete and continuous almost conservative systems.

These studies can be used to develop modern navigation and gyroscopic instruments that are used in aircraft industry and ship industry.

\section{References}

[1] Koshlyakov, V. N. (1972). Teorija giroskopicheskih kompasov. Moscow: Nauka, 344.

[2] Novytskyy, V. V., Zinchuk, M. O., Teteriatnyk, O. V. (2016). Stabilization and robust stability of continuous almost conservative systems. Vìsnik Zaporìz'kogo nacìnal'nogo unìversitetu. Fìziko-matematičnì nauki, 1, 184-191.

[3] Zinchuk, M. O., Novytskyy, V. V. (2007). Stability and stabilization linear parametric dynamic systems. Zbirnyk prats Instytutu matematyky Natsionalnoi akademii nauk Ukrainy, 4 (2), 58-71.

[4] Barnett, S., Cameron, R.G. (1985). Introduction to Mathematical Control Theory. Oxford: Clarendon press, 404.

[5] Zinchuk, M. O., Svyatovets, I. F., Teteriatnyk, O. V. (2016). On asymptotic solutions of matrix equations Lyapunov and Riccati for almost conservative systems. Visnyk Zaporizkoho natsionalnoho unyversytetu: Zbirnyk naukovykh prats. Fizyko-matematychni nauky, 2, 110-121.

[6] Novytskyy, V. V., (2004). Rivniannia Liapunova dlia maizhe konservatyvnykh system. NAN Ukrainy. Instytut matematyky, Kyiv, 7, 34.

[7] Prasolov, V. V. (1996). Zadachi i teoremy linejnoj algebry. Moscow: Nauka, 304.

[8] Zinchuk, M. O., Novytskyy, V. V. (2005). Investigation of the Lyapunov equation for discrete almost conservative systems. Analitychni doslidzhennia modelei mekhanichnykh system. Zbirnyk prats Instytutu matematyky Natsionalnoi akademii nauk Ukrainy, 8, 1-26.

[9] Teteriatnyk, O.V. (2014). Analytic expression of optimal control for systems of two coupled controllable almost conservative oscillators. Zbirnyk prats Instytutu matematyky Natsionalnoi akademii nauk Ukrainy, 11 (5), 231-239.

[10] Gantmaher, F. R. (2010). Matrix theory. Moscow: fizmatlit, 560.

[11] Lankaster, P. (1973). Matrix theory. Moscow: Nauka, 280.

[12] Horn, R. A., Johnson, C. R. (2012). Matrix analysis. Cambridge university press, 643. 